\title{
Fibonacci varieties
}

\section{Ann Chi Kim}

This is a report on a family of varieties of algebraic systems that are groups with an additional unary operation satisfying certain laws. The study was inspired by the theory of Fibonacci groups, and the free one-generator algebras of our varieties turn out to have, in fact, the abelianized Fibonnaci groups as their underlying groups.

\section{Introduction and preliminaries}

Conway [3] introduced a family of groups, now called "Fibonacci groups" and denoted by $F(2, n)$, with presentations

$$
F(2, n)=\operatorname{gp}\left(a_{0}, a_{1}, \ldots, a_{n-1} ; a_{i+2}=a_{i+1} a_{i}, i \bmod n\right) \text {. }
$$

These groups were studied in detail by Johnson, Wamsley, and Wright [5], and their paper also contains a brief history and bibliography of Fibonacci groups, as well as certain generalisations.

$F(2, n)$ has an obvious automorphism $\alpha$, say, of order $n$, which maps $a_{i}$ on $a_{i+1}(i \bmod n)$. In the present paper I study the effect of introducing such an automorphism as an additional algebraic operation, denoted by $\phi$, with laws to correspond to the above situation. Thus the set-up, in universal algebra terminology, is as follows.

Received 25 July 1978. The author thanks Emeritus Professor B.H. Neumann, who first introduced him to Fibonacci groups, for his continuing interest, for his readiness for discussion, for his mathematical optimism, and for help with preparing this paper for publication. The author also wishes to thank Dr C.M. Campbell and Dr D.L. Johnson for information they sent him on generalised Fibonacci groups. Finally, the author's gratitude is due to Professor R.C. Lyndon, who sent him an unpublished manuscript [6] on infinite Fibonacci groups. 
Our algebraic systems, or "algebras" for short, have a binary "multiplication" $\mu$, a unary "inversion" $\iota$, a nullary "neutral element" $e$, and a further unary operation $\phi$. However, we immediately simplify the notation by writing $x y$ for $x y \mu$ and $x^{-1}$ for $x \iota$, in accordance with usual group practice.

The basic laws are those of groups, which we do not list, plus those that make $\phi$ a group endomorphism:

$$
\left\{\begin{array}{c}
(x y) \phi=x \phi y \phi, \\
x^{-1} \phi=(x \phi)^{-1} \\
e \phi=e .
\end{array}\right.
$$

We further postulate the "Fibonacci law"

$$
x \phi^{2}=x \phi x,
$$

and the law that gives $\phi$ finite order $n$ :

$$
x \phi^{n}=x \text {. }
$$

This last law ensures that $\phi$ is invertible, and thus acts as an automorphism on the underlying group. We write $\phi^{-1}$ for the inverse (which equals $\phi^{n-1}$ ) of $\phi$. We denote by $\mathrm{V}_{n}$ the variety defined.as above. If $A$ is an algebra in $\stackrel{V}{n}_{n}$, we denote its underlying group by A

We first show that $A_{0}$ is an abelian group.

LEMMA. If $\mathrm{A}$ is an algebra in $\underline{\mathrm{V}}_{n}$, then the underlying group $\mathrm{A}_{0}$ is abetian.

Proof. The laws (1), (2) imply the laws

$$
\begin{aligned}
& (x y) \phi^{2}=(x y) \phi x y=x \phi y \phi x y, \\
& (x y) \phi^{2}=x \phi^{2} y \phi^{2}=x \phi x y \phi y ;
\end{aligned}
$$

whence

$$
x \phi y \phi x y=x \phi x y \phi y .
$$


The group laws simplify this to

$$
y \phi x=x y \phi,
$$

and, putting $y=z \phi^{-1}$, so that $y \phi=z$,

$$
z x=x z \cdot \quad / /
$$

\section{The monogenic free algebras}

We now consider the monogenic (that is one-generator) free algebra $G(n)$, say, of $\mathrm{V}_{n}$; let $a$ be a free generator of it, and put

$$
a=a_{0}, \alpha \phi=a_{1}, \ldots, a \phi^{i}=a_{i} .
$$

The law (3) ensures that $a_{n}=a_{0}, a_{n+1}=a_{1}$, and so on. Thus the underlying group $G_{0}(n)$ is generated, as group, by $\left\{a_{0}, a_{1}, \ldots, a_{n-1}\right\}$. Now the law (2) ensures that

$$
a_{i+2}=a_{i+1} a_{i}, i \bmod n \text {. }
$$

Thus $G_{0}(n)$, in terms of the generators $a_{0}, a_{1}, \ldots, a_{n-1}$, satisfies the defining relations of $F(2, n)$, and thus must be an epimorphic image of $F(2, n)$. We know already, from the lemma, that $G_{0}(n)$ is abelian; hence it must, in fact, be isomorphic to a factor group of $A(2, n)=F(2, n) / F^{\prime}(2, n)$, the "abelianized Fibonacci group", $F^{\prime}(2, n)$ being the commutator subgroup of $F(2, n)$, and the notation $A(2, n)$ being that of Johnson, Wamsley, and Wright [5].

We now prove that $G_{0}(n)$ is, in fact, isomorphic to this abelianized Fibonacci group.

THEOREM. The group $G_{0}(n)$ underlying the monogenic free algebra $G(n)$ of the variety $\stackrel{\mathrm{V}}{\rightarrow}$ is isomorphic to $A(2, n)$.

Proof. As we know already that $G_{0}(n)$ is an epimorphic image of $A(2, n)$, it suffices to show that, with the obvious definition of the action of $\phi$ on $A(2, n)$, the laws of $\underline{v}$ are satisfied. The action of $\phi$ is to be that induced on $A(2, n)$ by the automorphism $\alpha$ of $F(2, n)$. 
This clearly defines then an automorphism of $A(2, n)$, and this automorphism has order $n$. Thus we need only verify the fact that the law (2) is satisfied, not only by the generators $a_{0}, a_{1}, \ldots, a_{n-1}$, but by all elements of our group. But this follows immediately from the fact that our group is abelian; for, if we know that for arbitrary group elements $g, h$, say, the relations

$$
g \phi^{2}=g \phi g, h \phi^{2}=h \phi h
$$

hold, then also

$$
(g h) \phi^{2}=g \phi^{2} h \phi^{2}=g \phi g h \phi h=g \phi h \phi h=(g h) \phi g h ;
$$

that is to say, the corresponding relation holds for the product $g h$. Similarly

$$
\left(g^{-1}\right) \phi^{2}=\left(g \phi^{2}\right)^{-1}=(g \phi g)^{-1}=g^{-1}(g \phi)^{-1}=(g \phi)^{-1} g^{-1}=\left(g^{-1}\right) \phi g^{-1} ;
$$

thus the corresponding relation also holds for inverses. As it holds for the generators, it then holds throughout the group. //

We summarize below the structure of $A(2, n)=G_{0}(n)$; this appears to have been first determined by Bumby (quoted in [8], where, however, fewer details are given, and where, moreover, a slight error has crept in: $n \equiv 0(\bmod 2)$ should read $n \equiv 2(\bmod 4))$. The description uses the well-known Fibonacci sequence $\left\{f_{i}\right\}$ defined by

$$
f_{i+2}=f_{i+1}+f_{i}, f_{0}=0, f_{1}=1 .
$$

The order of $A(2, n)$ is then $g_{n}$, where

$$
g_{n}=f_{n-1}+f_{n+1}-1-(-1)^{n}
$$

the sequence $\left\{g_{i}\right\}$ can also be defined by the recurrence

$$
g_{i+3}=2 g_{i+1}+g_{i}+2, g_{0}=0, g_{1}=1, g_{2}=1 \text {. }
$$

$A(2, n)$ is the direct product of two cyclic groups, of orders $p$ and $q$, say, where $p$ divides $q$; that is

$$
A(2, n)=c_{p} \times c_{q}, p q=g_{n} .
$$


If $p=1$, then $A(2, n)$ is itself cyclic. This is the case if, and only if, $n$ is odd and not divisible by 3 ; that is $(n, 6)=1$. If $n$ is odd and a multiple of 3 , that is $n \equiv 3(\bmod 6)$, then

$$
p=2, q=\frac{1}{2}\left(f_{n-1}+f_{n+1}\right)=\frac{1}{2} g_{n} .
$$

If $n$ is even, but not divisible by 4 , that is $n=2(\bmod 4)$, then

$$
p=q=f_{\frac{1}{2} n-1}+f_{\frac{l^{2} n+1}{}}=\left(g_{n}\right)^{\frac{1}{2}} \text {. }
$$

Finally, if $n$ is divisible by 4 , then

$$
p=f_{\frac{1}{2} n}=\left(\frac{1}{5} g_{n}\right)^{\frac{1}{2}}, q=5 p .
$$

The following table summarizes these results.

$\begin{array}{ccc}n(\bmod 12) & p & q \\ 0 & f_{\frac{1}{2} n} & 5 f_{\frac{1}{2} n} \\ 1 & 1 & g_{n} \\ 2 & f_{\frac{1}{2} n-1}+f_{\frac{1}{2} n+1} & f_{\frac{1}{2} n-1}+f_{\frac{1}{2} n+1} \\ 3 & 2 & \frac{1}{2}\left(f_{n-1}+f_{n+1}\right) \\ 4 & f_{\frac{1}{2} n} & 5 f_{\frac{1}{2} n} \\ 5 & 1 & g_{n} \\ 6 & f_{\frac{1}{2} n-1}+f_{\frac{1}{2} n+1} & f_{\frac{1}{2} n-1}+f_{\frac{3}{2} n+1} \\ 7 & 1 & g_{n} \\ 8 & f_{\frac{1}{2} n} & 5 f_{\frac{1}{2} n} \\ 9 & 2 & f_{\frac{1}{2}}\left(f_{n-1}+f_{n+1}\right) \\ 10 & f_{\frac{1}{2} n-1}+f_{\frac{1}{2} n+1} & f_{\frac{1}{2} n-1}+f_{\frac{3}{2} n+1} \\ 11 & 1 & g_{n}\end{array}$

It is clear that a number of generalizations can be investigated, and I hope to discuss some of them in a later paper.

The references below list relevant source materials in addition to references quoted in the text. 


\section{References}

[1] A.M. Brunner, "Fibonacci sequences and group theory" (PhD thesis, Australian National University, Canberra, 1973). See also: Abstract, Bull. Austral. Math. Soc. 9 (1973), 473-474.

[2] C.M. Campbell and E.F. Robertson, "Deficiency zero groups involving Fibonacci and Lucas numbers", Proc. Roy. Soc. Edinburgh Sect. A (to appear).

[3] J.H. Conway, "Advanced problem 5327", Amer. Math. Monthly 72 (1965), 915.

[4] D.L. Johnson, Presentations of groups (London Mathematical Society Lecture Notes Series, 22. Cambridge University Press, Cambridge, London, New York, Melbourne, 1976).

[5] D.L. Johnson, J.W. Wamsley, and D. Wright, "The Fibonacci groups", Proc. London Math. Soc. (3) 29 (1974), 577-592.

[6] Roger C. Lyndon, "On a family of infinite groups introduced by Conway", unpublished manuscript.

[7] B.H. Neumann, "The reflection principle", Math. Chronicle 5 (1976), $1-7$.

[8] Various Authors, "Generators and relations for cyclic groups" (Solution to advanced problem 5327, see [3] above), Amer. Math. lonthly 74 (1967), 91-93.

Department of Mathematics,

Busan National University,

Busan,

Korea. 